\title{
The spatial distribution of attention following an exogenous cue
}

\author{
JOHN M. HENDERSON and ANDREW D. MACQUISTAN \\ University of Alberta, Edmonton, Alberta, Canada
}

\begin{abstract}
Three target-discrimination experiments were conducted to explore the spatial distribution of covert visual attention following an exogenous cue. On each trial, a brief peripheral onset was followed by a target stimulus in an otherwise empty visual field at one of eight (Experiment 1) or one of four (Experiments 2 and 3) possible locations centered at the fixation point. The spatial relation between the cue and the target was manipulated. The main results were that (1) performance was better at the cued location than at another nearby location in the same visual quadrant; (2) performance was not affected by the major horizontal and vertical visual meridians; and (3) performance was affected by the spatial distance between the cued and target locations. Together, the results suggest that the spatial distribution of exogenously oriented attention can be most easily integrated with a simple gradient model.
\end{abstract}

A central issue in the study of visual cognition is the human observer's ability to select a particular stimulus in the visual field for further processing and for action. Often, this selection is overt, such as when eye and head movements bring the selected stimulus to the foveae. As has been known for over 100 years, the human observer can also select a stimulus location for further processing through the covert deployment of visual attention (e.g., see James, 1890, for quotations and a discussion of Helmholtz's observations). More recently, a great deal of effort has been expended in the attempt to understand the properties of covert visual attention and the mechanisms by which it is allocated.

An important question in the study of covert visual attention is the manner in which attention is spatially distributed within the visual field. Two current popular models of attentional allocation, the zoom-lens (Eriksen \& St. James, 1986; Eriksen \& Webb, 1989; Eriksen \& Yeh, 1985) and gradient (Downing, 1988; Downing \& Pinker, 1985; Henderson, 1991; LaBerge \& Brown, 1989; Madden, 1992; Shulman, Wilson, \& Sheehy, 1985) models, can be considered instantiations of a more general specific-location hypothesis. According to this hypothesis, visual attention can be directed to a highly restricted region of the visual field. In contrast, a number of theorists have proposed versions of what we will call the general-region hypothesis. Perhaps the most prominent instantiation of the general-region hypothesis is the meridian boundary model proposed by Hughes and Zimba (1985, 1987). According to this model, attention must be

The research reported in this article was supported by the Natural Sciences and Engineering Research Council of Canada. We would like to thank Ray Klein and two anonymous reviewers for their thoughtful comments. Correspondence concerning this article can be addressed to J. M. Henderson, Department of Psychology, 129 Psychology Research Building, Michigan State University, East Lansing, MI 48824. directed to large regions of the visual field and cannot be directed to restricted, specific locations. Attentional effects are produced by activation of an attended and/or suppression of an unattended region, where regions are visual hemifields (Hughes \& Zimba, 1985) or visual quadrants (Hughes \& Zimba, 1987) bounded by and defined in terms of the vertical and/or horizontal visual meridians.

The zoom-lens and gradient models of attentional deployment were both originally proposed to account for the results of the many studies that engage the endogenous attentional system. The endogenous system can be characterized as that visual-attentional system that is centrally or consciously controlled. This system is generally manipulated experimentally through instructional set (e.g., LaBerge \& Brown, 1989) or symbolic location cues that are probabilistically valid (e.g., Eriksen \& Yeh, 1985; Posner, 1980; Posner, Snyder, \& Davidson, 1980). In contrast, relatively less effort has been expended on the attempt to explore the spatial distribution of attention when the exogenous attentional system is engaged. The exogenous system is engaged by abrupt visual onsets in the visual field (Jonides, 1981; Yantis \& Jonides, 1984). These two systems appear to have some distinctive characteristics. For example, the exogenous and endogenous systems respectively appear to produce relatively fast but brief versus slow but sustained response profiles (Müller \& Rabbitt, 1989; Nakayama \& Mackeben, 1989), relatively large versus small effect sizes (Jonides, 1981; Müller \& Rabbitt, 1989; Nakayama \& Mackeben, 1989), inhibition of return at the cued location versus no such effect (Maylor, 1985; Posner \& Cohen, 1984; Rafal, Calabresi, Brennan, \& Sciolto, 1989), and a relationship to the eye-movement programming system versus no such relationship (Rafal et al., 1989).

A central purpose of the current article is to examine the extent to which the specific-location hypothesis characterizes the spatial distribution of visual attention when the 
exogenous system is engaged. Distinguishing between the specific-location and general-region hypotheses of attention is important in specifying the function of exogenous visual attention in vision. For example, in many recent models, visual attention is the "glue" used to solve the "binding problem" of determining which visual features go with which others in defining an object (Briand \& Klein, 1987; Treisman \& Gelade, 1980). If attention as studied in cuing paradigms cannot be narrowed down to the location of a specific object, then the role of attention in solving the binding problem would be called into question (see, e.g., Tsal, 1989). Similarly, it has been suggested that visual attention allows selection of specific locations in the visual field for action. For example, recent models of eye-movement control suggest that directing visual attention to a specific location provides targeting information for a subsequent saccade (Henderson, 1988, 1992; Henderson \& Ferreira, 1990; Henderson, Pollatsek, \& Rayner, 1989; Morrison, 1984; Pollatsek \& Rayner, 1990). One way to think about this process is that attention allows binding of visual coordinates to motor coordinates (Henderson, in press). Again, if attention cannot be directed toward a specific location, it would be difficult to argue that spatial attention plays a major role in selection for action. In the following experiments, we explored the spatial distribution of attention in an exogenous attentional paradigm. It is important to understand the nature of the spatial distribution of attention when the exogenous attentional system is engaged, because it appears that the exogenous system is more involved than the endogenous system in both feature binding (Briand \& Klein, 1987; Klein, Kingstone, \& Pontefract, 1992) and eye-movement control (Rafal et al., 1989).

In our experiments, we sought to examine three particular issues. First, we attempted to distinguish between the specific-location and general-region approaches to visual attention by determining whether exogenous visual attention can be allocated to a specific location in the visual field, or whether attention instead must be allocated to a larger region defined by the visual meridians. In our experiments, we presented a target stimulus in an otherwise empty field, because Hughes and Zimba (1987) have suggested that apparent specific-location effects are due to confounds arising from the presence of distractors in the visual field.

Second, if it were the case that attention could be allocated to a specific location, we wanted to determine whether there would be any effect at all of having to cross a visual meridian from cued location to target location in an exogenous cuing paradigm. An effect of visual meridian overlayed upon a specific-location effect would appear more likely than an effect of visual meridian alone. Previous studies employing an endogenous paradigm similar to that used by Hughes and Zimba (1987), in which subjects detect a luminance increment, have observed an effect of specific attentional allocation combined with a meridian effect (e.g., Downing \& Pinker, 1985; Rizzolatti, Riggio, Dascola, \& Umiltà, 1987; Tassinari,
Aglioti, Chelazzi, Marzi, \& Berlucchi, 1987). In addition, there are neurophysiological reasons, such as the presence of the two cortical hemispheres, to expect that there might be at least a horizontal meridian effect (see, e.g., Reuter-Lorenz, Kinsbourne, \& Moscovitch, 1990). On the other hand, several recent attempts to find such an effect in exogenous attention paradigms have been unsuccessful (Egly \& Homa, 1991; Henderson, 1991).

Third, if attention were directed to a specific location, would performance for targets at uncued locations fall off gradually with distance from the cued location, or would performance at uncued locations be equivalent at all distances? This issue is central to the distinction between the zoom-lens and gradient models of attentional allocation. Both models assume that visual attention is a limited resource system that can be directed to spatial regions of varying size, including relatively specific regions. According to the zoom-lens model, the attended region is discretely bounded (with a small gradient fringe; see Eriksen \& Webb, 1989), whereas according to the gradient model, attention falls off gradually from the center of the attended region as a function of spatial distance.

In the current experiments, attention was manipulated through the use of an abrupt, transient onset cue in the visual periphery. We examined performance for a stimulus that appeared at the cued location versus some other location. On each trial, a target stimulus (an X or an $\mathrm{O}$ ) appeared in an otherwise empty field at one of eight (Experiment 1) or one of four (Experiments 2 and 3) locations equidistant from each other and from fixation. Prior to target presentation, a location cue briefly appeared. The target then appeared either at the cued location or at some other uncued location. The subject's task following presentation of the target was to execute a forced-choice response indicating whether an $\mathrm{X}$ or an $\mathrm{O}$ had appeared on that trial.

The present study is methodologically and procedurally similar to two previous studies of the effects of visual meridians and spatial distance on target discrimination performance following exogenous cues (Egly \& Homa, 1991; Henderson, 1991; see also Klein \& McCormick, 1989, for a similar set of experiments in which endogenous cues were used). Egly and Homa found that spatial distance from the cued location predicted performance in their target discrimination task. Further, they found no effect of the visual meridian. However, Egly and Homa employed response time as their dependent measure. In the following experiments, we examined the influence of visual meridians and spatial distance on both response accuracy (Experiments 1 and 2) and response time (Experiment 3). In addition, Egly and Homa could not determine whether their effects were primarily due to costs or to benefits, because they did not employ a neutral condition. This issue is important, given that Hughes and Zimba (1985, 1987) have claimed that cuing effects are primarily due to inhibition at uncued regions. In the following experiments, we included a neutral condition in order to determine whether cuing effects in an exogenous paradigm 
produce both costs and benefits. Finally, in Egly and Homa's study, the target appeared at the cued location with a higher probability than that for an uncued location. Because of this difference in target location probability, subjects may have been induced to engage the endogenous attentional system. In the present experiments, the target was equiprobable at the cued and uncued locations so that subjects would have no reason to attend endogenously.

In Henderson's (1991) study, it was found that performance for invalid targets that appeared outside of the visual quadrant in which the cue had been shown was poorer than performance for within-quadrant invalid targets. In those experiments, however, the within-quadrant invalid targets were spatially closer to the cued location than the outside-quadrant invalid targets were. Therefore, it was impossible to determine whether the advantage for the within-quadrant over the outside-quadrant invalid targets was due to the additional spatial distance, or to the visual meridian that separated the cued and target locations in the latter but not the former case. The current experiment allowed an examination of this issue because all target locations were equidistant from each other.

\section{EXPERIMENT 1}

In Experiment 1, we explored several issues related to covert exogenous attentional orienting. First, can attention be allocated to a specific location rather than to a general region of the visual field? Second, when a target appears at an uncued location, is discrimination performance for that target affected by whether the target and cue are separated by a visual meridian? Third, is discrimination performance for a target at an uncued location affected by the spatial distance of the target from the cued location?

In order to explore these issues, we compared performance on targets appearing at the cued location (valid condition); at an uncued location within the quadrant containing the cue (within-quadrant invalid); at an uncued location outside the quadrant containing the cue, but at the same distance from the cue as that of the withinquadrant invalid target (outside-quadrant invalid); and at an uncued location diagonally opposite the cued location (diagonal invalid). In addition, a neutral condition consisting of all eight cues simultaneously displayed was included to allow an assessment of costs and benefits in the valid and invalid conditions.

\section{Method}

Subjects. Twelve University of Alberta undergraduate students participated for credit toward their introductory psychology class. All subjects had normal or corrected-to-normal vision.

Apparatus and Stimuli. The stimuli were presented in black on white on a high-resolution color video monitor placed $35 \mathrm{~cm}$ from the subject. A chin- and forehead rest was used to maintain viewing distance. The target stimuli were the capital letters $\mathrm{X}$ and $\mathrm{O}$ created in a $7 \times 12$ pixel matrix $1^{\circ}$ of visual angle high and $44^{\prime}$ wide. The spatial masking stimulus consisted of the same two characters superimposed. The location cue was an underline $15^{\prime}$ high and $1^{\circ} 20^{\prime}$ wide. The distance from the bottom of the target position to the top of the cue was $30^{\prime}$.

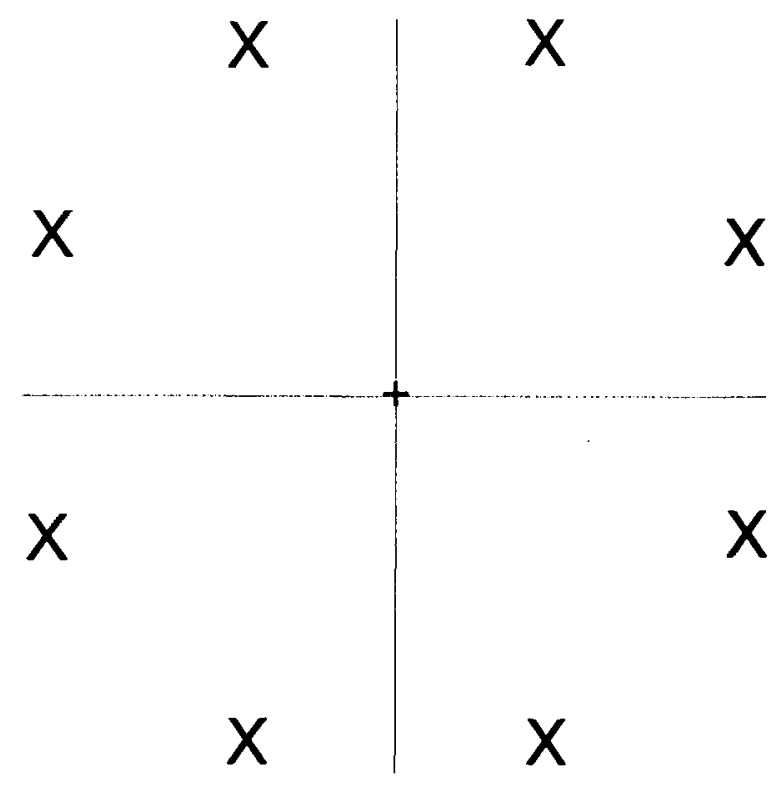

Figure 1. Schematic illustration of the stimulus layout in Experiment 1. The eight possible target locations are indicated (by Xs) in relation to the horizontal and vertical visual meridians (lines, which were not displayed).

The stimuli could be displayed at eight locations around an imaginary circle centered at the point of fixation. The eight locations were arranged so that two locations appeared in each quadrant of the visual field (see Figure 1). The center of each target location was $9^{\circ} 41^{\prime}$ from the fixation point. Target locations were equidistant from each other, with about $7^{\circ} 14^{\prime}$ center to center.

The subjects responded by pressing the appropriate microswitch located on a table-mounted response panel. The response panel was interfaced with a dedicated I/O board; pressing a button on the response panel generated a system interrupt and stopped a millisecond clock. Stimulus presentation and response collection were controlled by an 80286-based microcomputer.

Procedure. The subjects were asked to make a judgment regarding the identity of a target stimulus viewed peripherally. Each trial began with the presentation of a central fixation cross along with the mask stimulus indicating the eight possible target locations. When the subject was ready, he or she pushed a button to start the trial. The central fixation cross was then displayed alone for $1,000 \mathrm{msec}$. A location cue then appeared for $100 \mathrm{msec}$, followed by a target stimulus ( $\mathrm{X}$ or $\mathrm{O}$ ) for $50 \mathrm{msec}$. A spatial mask followed the target and remained on the screen until the subject responded. The mask consisted of a superimposed $X$ and $O$ at each of the eight possible target positions. Each display immediately followed the preceding display (i.e., 0-msec interdisplay interval), and the stimulus on the preceding display was extinguished with the onset of the next display (i.e., the location cue was removed with the onset of the target), although the fixation cross remained visible throughout the trial. The subject executed a forced-choice response by pressing one of two response keys as quickly as possible following target onset while maintaining accuracy. After the response, there was a 2,000 -msec intertrial interval.

At the beginning of a session, subjects were informed about the general aspects of the procedure. The rapid nature of the visual events was discussed, and the subjects were encouraged to pay careful attention to the display on each trial. The subjects were informed that the events occurring on the screen were too rapid for eye movements to help, and they were therefore encouraged to maintain 
fixation at the center of the screen. The first few practice trials convinced the subjects that maintaining fixation was the best strategy. (When subjects are induced to move their eyes as quickly as possible, the minimum latency plus duration for a $7^{\circ}$ saccade is greater than the 150-msec total display duration used in the present experiment; see, e.g., Abrams, Meyer, \& Kornblum, 1989; Henderson, Pollatsek, \& Rayner, 1987; Rayner, Slowiaczek, Clifton, \& Bertera, 1983).

The exogenous cues in the present experiment were uninformative about the location of the target. Given a location cue (rather than the neutral cue), the target appeared at the cued location with .25 probability, at the within-quadrant invalid location with .25 probability, at the outside-quadrant invalid location with .25 probability, and at the diagonal invalid location with .25 probability. Given the neutral cue, the target appeared at any of the eight possible locations with an equal probability of .125 . The experiment contained 240 trials broken down as follows: 8 (target locations) $\times 5$ (cue conditions) $\times 2$ (target type) $\times 3$ (replications) .

Each session began with the instructions along with several example trials, followed by 32 practice trials and one test block. The entire experiment lasted about $45 \mathrm{~min}$

\section{Results}

Because of the brief presentation of the targets and the resulting relatively high error rates, percentage correct was considered the primary dependent measure. However, analyses were conducted on both mean percentages correct and mean response times for correct responses.

Percentage correct. Table 1 presents the mean accuracy rates as a function of cue condition. A one-way omnibus analysis of variance showed a reliable effect of cuing $\left[F(4,44)=16.3, M S_{\mathrm{e}}=.0088, p<.001\right]$.

The main question addressed in this experiment was whether the major visual meridians play a role in attentional orienting following exogenous cues. First, we examined whether performance would be better in the valid condition than in the inside-quadrant invalid condition. If attention can only be allocated to a visual quadrant defined by the major visual meridians, performance should be equivalent in these conditions. If, on the other hand, attention can be allocated to a specific location, performance in the valid condition should be better than performance in the invalid-within condition. There was a $12 \%$ advantage for the valid condition over the withinquadrant invalid condition $\left[F(1,11)=17.3, M S_{\mathrm{e}}=.0105\right.$, $p<.005]$, which was consistent with the specific-location hypothesis. Second, we asked whether the need to cross a visual meridian following an invalid attentional cue

Table 1

Mean Percentage Correct, Mean Response Time (in Milliseconds), and Costs and Benefits in Relation to the Neutral Condition in Experiment 1

\begin{tabular}{lccccc}
\hline & \multicolumn{2}{c}{ \% Correct } & & \multicolumn{2}{c}{ RT } \\
\cline { 2 - 3 } \cline { 5 - 6 } \multicolumn{1}{c}{ Condition } & $M$ & Cost/Benefit & & $M$ & Cost/Benefit \\
\hline Valid & 91 & $8^{*}$ & & 656 & $90 \dagger$ \\
Within-quadrant invalid & 78 & $-5^{*}$ & & 773 & -27 \\
Outside-quadrant invalid & 78 & $-5^{*}$ & & 766 & -20 \\
Diagonal invalid & 70 & $-13 \dagger$ & & 870 & $-124^{*}$ \\
Neutral & 83 & & & 746 & \\
\hline
\end{tabular}

Note-Positive numbers indicate benefits and negative numbers indicate costs in relation to the neutral condition. ${ }^{*} p<.05 . \quad+p<.01$. would lead to poorer performance than the case in which it would not be necessary to cross a meridian; to do this, we compared performance in the within-quadrant invalid trials with performance in the outside-quadrant invalid trials. The observed difference between these two conditions was less than $1 \%$ and not reliable $[F(1,11)<1$, $\left.M S_{\mathrm{e}}=.0026\right]$.

It might be argued that the need to cross a meridian following an invalid cue would vary, depending on whether the meridian was horizontal or vertical. For example, it could be that crossing the vertical meridian is more difficult because it involves switching processing from one cerebral hemisphere to the other (Kinsbourne, 1987). In order to test this possibility, we divided the outsidequadrant invalid trials into two subconditions, those that involved crossing the vertical meridian and those that involved crossing the horizontal meridian. A simple contrast indicated that the apparent $3.5 \%$ difference in performance between crossing the vertical $(76 \%)$ and crossing the horizontal $(79.5 \%)$ meridian did not approach significance $\left[F(1,11)<1, M S_{\mathrm{e}}=.0154\right]$.

The costs and benefits associated with each of the other primary cue conditions in relation to the neutral condition are shown in Table 1. Performance in the neutral condition was roughly intermediate between performance in the valid and invalid conditions, suggesting that a valid cue led to benefits in processing while an invalid cue led to costs.

Response time. Response time analyses included only correct trials. In addition, outlier response times less than $100 \mathrm{msec}$, greater than $3,000 \mathrm{msec}$, or more than $3 \mathrm{stan}$ dard deviations from the cell mean for that condition and that subject were discarded to reduce variability. In total, $4.0 \%$ of the potential data points were categorized as outliers.

Table 1 presents mean response times as a function of cue condition. As in the accuracy data, there was a reliable overall effect of cue type $\left[F(4,44)=8.99, M S_{e}=\right.$ $15,528, p<.001]$. The advantage was $117 \mathrm{msec}$ for targets appearing at the cued location over targets appearing at an uncued location in the same quadrant $[F(1,11)=$ $\left.12.4, M S_{\mathrm{e}}=13,049, p<.005\right]$, suggesting that attention is directed to a specific location. In the test for an effect of crossing a meridian when the cue was invalid, the difference between the within-quadrant and outsidequadrant invalid conditions was a small and nonsignificant $7 \mathrm{msec}\left[F(1,11)<1, M S_{\mathrm{e}}=2,472\right]$.

We again divided the outside-quadrant invalid condition into two subconditions, those that involved crossing the vertical meridian and those that involved crossing the horizontal meridian, in order to determine whether there would be a differential effect of meridian type. There was a marginally reliable tendency for the response times to be longer when the target appeared across the vertical meridian (792 msec) compared with the horizontal meridian $(731 \mathrm{msec})\left[F(1,11)=4.17, M S_{\mathrm{e}}=10,556, p=.06\right]$.

The costs and benefits associated with each of the other cue conditions in relation to the neutral condition are shown in Table 1. Response times in the neutral condi- 
tion were roughly intermediate between performance in the valid and invalid conditions, which is consistent with the accuracy data.

\section{Discussion}

The results of the present experiment support several general conclusions. First, targets appearing at a validly cued location were discriminated more accurately (and more rapidly) than were targets appearing at an uncued location. These data clearly show that an unpredictive exogenous cue exerts an influence on target discrimination. Second, performance at the cued location was better than performance at an uncued location within the same quadrant, providing evidence that attention can be allocated to a specific location rather than only to regions defined by the major visual meridians. Third, when the cue was invalid, targets that appeared outside of the cued quadrant were just as accurately (and rapidly) discriminated as were targets inside the cued quadrant, given that distance from the cue was held constant. Thus, it does not appear that crossing a meridian necessarily has a detrimental effect on attentional allocation or reallocation. Fourth, targets appearing at the cued location were discriminated more accurately (and more rapidly), whereas targets appearing at an uncued location were discriminated less accurately (and less rapidly) than were targets following a neutral cue. Thus, the exogenous cues in this experiment produced both benefits and costs for target discrimination. Fifth, in the single-cue (i.e., non-neutral) conditions, performance was most accurate (and fastest) at the cued location, followed by the closer invalid locations (insideand outside-quadrant invalid), and worst at the farthest invalid location (diagonal invalid). These results suggest that attention drops off continuously as distance from the cued location increases, a finding that supports gradient models of attentional allocation (e.g., those of Downing \& Pinker, 1985; Henderson, 1991; LaBerge \& Brown, 1989; Shulman et al., 1985).

As noted above, performance for invalidly cued targets was equivalent at within-quadrant and outside-quadrant locations in this experiment. This result contrasts with the results of Henderson (1991), who found that withinquadrant performance was better than outside-quadrant performance in two out of the three experiments in which it was tested. However, one major difference between the present experiment and those of Henderson is that in the latter, the distance between the cued location and the within-quadrant location was smaller than the distance between the cued location and the outside-quadrant location. In the present experiment, the distances between the cued location and the two nearby locations were equated. Therefore, the data across the two studies also support a gradient model suggesting that attention drops off continuously from the cued location as distance from the cue increases.

We note that one finding in the present experiment may be seen as supporting theories that predict a meridian effect. This finding is that response times for targets that appeared across the vertical meridian from the cued lo- cation were (marginally) $61 \mathrm{msec}$ slower than response times for targets that appeared across the horizontal meridian. This finding is consistent with Hughes and Zimba (1987), who found greater inhibition across the vertical meridian from the cued location in an endogenous attention paradigm. However, we believe that our data should be viewed with caution for two reasons. First, these means are based on conditions in which over $20 \%$ of the data points were excluded because of response errors. The primary dependent measure was response accuracy, and there was no hint of a reliable difference on that measure. Second, when the outside-quadrant invalid condition is divided into across-vertical and across-horizontal subconditions, the positions of the cues and targets are no longer held constant. Instead, the across-vertical subcondition involves cues and targets that appear at the top and bottom of the display, whereas the across-horizontal condition involves cues and targets at the sides of the display. (We note that none of the main conditions suffer from this problem.) Because letter recognition is known to be more accurate farther into the periphery horizontally compared with vertically from fixation (see, e.g., Bouma, 1971), the apparent meridian effect may be artifactual. In other words, the apparent meridian effect may actually be an acuity effect. Experiments 2 and 3 were attempts to explore the potential difference between the vertical and horizontal meridians by using a display that equated cue and target locations regardless of the meridian crossed in the outside-quadrant invalid condition.

\section{EXPERIMENT 2}

In Experiment 2, we directly tested whether presenting a target at an invalid location that is across the vertical visual meridian from the cue leads to poorer performance than does presenting a target at an invalid location that is across the horizontal visual meridian. Cues and targets therefore appeared at four possible locations at the corners of an imaginary square centered at the point of fixation. We compared performance on targets appearing at the cued location (valid condition), at an uncued location that was across the vertical meridian but not across the horizontal meridian from the cued location (across-vertical invalid), at an uncued location that was across the horizontal but not across the vertical meridian from the cued location (across-horizontal invalid), and at an uncued location that was across both the vertical and the horizontal meridians (diagonal invalid). A neutral condition consisting of all four cues displayed simultaneously was also included to allow assessment of costs and benefits.

\section{Method}

Subjects. Twelve University of Alberta undergraduate students participated for credit toward their introductory psychology class. All subjects had normal or corrected-to-normal vision. None of the subjects had participated in Experiment 1.

Apparatus and Stimuli. The apparatus and stimuli were the same as those in Experiment 1, with the following exceptions. The stimuli could be displayed at four locations at the corners of an imaginary 


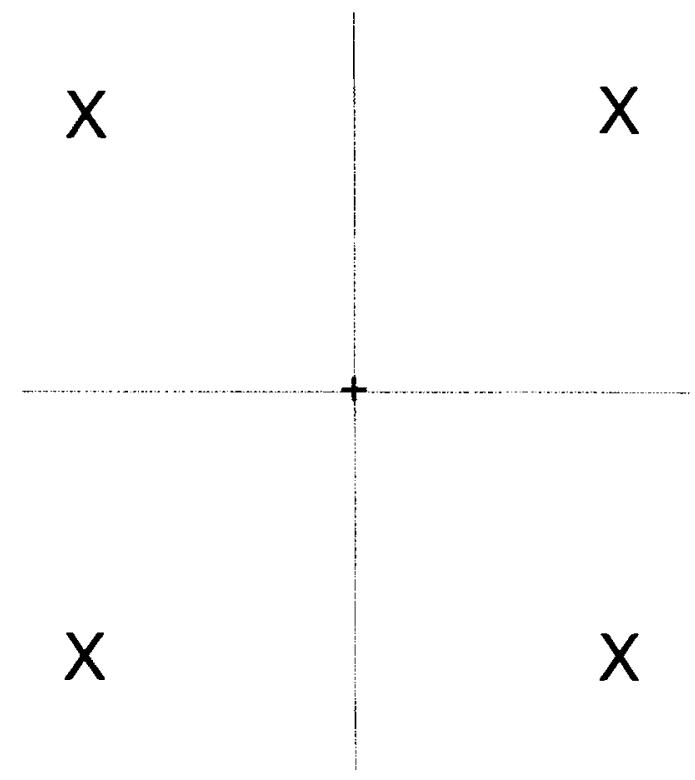

Figure 2. Schematic illustration of the stimulus layout in Experiments 2 and 3 . The four possible target locations are indicated (by $\mathrm{Xs)}$ in relation to the horizontal and vertical visual meridians (lines, which were not displayed).

square centered at the point of fixation. The four locations were arranged so that one location appeared in each quadrant of the visual field (see Figure 2). The center of each target location was $9^{\circ} 15^{\prime}$ from the fixation point. Target locations were equidistant from each other, with $12^{\circ} 13^{\prime}$ center to center. Stimulus presentation and response collection were controlled by an 80386 -based microcomputer.

Procedure. The procedure was identical to that used in Experiment 1 , except that only four potential target locations were employed. All timing and display parameters remained the same as in Experiment 1. The exogenous cues were again uninformative about the location of the target. Given a location cue (rather than the neutral cue), the target appeared at the cued location with .25 probability, at the across-horizontal invalid location with .25 probability, at the across-vertical invalid location with .25 probability, and at the diagonal invalid location with .25 probability. Given the neutral cue, the target appeared at each of the four possible locations with an equal probability of .25 . The experiment contained 160 trials broken down as follows: 4 (target locations) $\times 5$ (cue conditions) $\times 2$ (target type) $\times 4$ (replications)

Each session began with the instructions along with several example trials, followed by 32 practice trials and one test block. The entire experiment lasted under $30 \mathrm{~min}$.

\section{Results}

Because of the brief presentation of the targets and the resulting relatively high error rates, percentage correct was considered the primary dependent measure. However, analyses were conducted on both mean percentages correct and mean response times for correct responses.

Percentage correct. Table 2 presents the mean accuracy rates as a function of cue condition. A one-way omnibus analysis of variance showed a reliable effect of cuing $\left[F(4,44)=9.70, M S_{\mathrm{e}}=.0128, p<.001\right]$. As can be seen in Table 2, performance was better when the target appeared at the cued location than when it appeared at any of the uncued locations. This result replicates the finding from Experiment 1 and our previous experiments (Henderson, 1991).

The main question addressed in this experiment was whether performance would be worse in the acrossvertical invalid condition compared with the acrosshorizontal invalid condition, as suggested by a hemifield activation account of attentional allocation (Hughes \& Zimba, 1985). As can be seen in Table 2, performance in these two invalid conditions was identical at $62 \%$ correct $\left(F<1, M S_{\mathrm{e}}=.0049\right)$.

The costs and benefits associated with each of the other cue conditions in relation to the neutral condition are also shown in Table 2. Performance in the neutral condition was roughly intermediate between performance in the valid and invalid conditions, suggesting that a valid cue led to benefits in processing while an invalid cue led to costs.

Response time. Response time analyses included only correct trials. In addition, outlier response times less than $100 \mathrm{msec}$, greater than $3,000 \mathrm{msec}$, or more than $3 \mathrm{stan}$ dard deviations from the cell mean for that condition and that subject were discarded in order to reduce variability. In total, $1.3 \%$ of the potential data points were categorized as outliers.

Table 2 presents the mean response time as a function of cue condition. As in the accuracy data, there was a reliable effect of cue type $\left[F(4,44)=5.31, M S_{\mathrm{e}}=\right.$ $20,981, p<.005]$. In contrast to the accuracy data, there was a numerical response time disadvantage of $38 \mathrm{msec}$ for the across-vertical as opposed to the across-horizontal invalid condition. However, this difference did not approach significance $\left(F=1.11, M S_{\mathrm{e}}=16,155, p>.30\right)$.

The costs and benefits associated with each of the other cue conditions in relation to the neutral condition are shown in Table 3. Response times in the neutral condition were roughly intermediate between performance in the valid and invalid conditions, which is consistent with the accuracy data.

\section{Discussion}

The main question addressed in this experiment was whether crossing the vertical meridian would lead to poorer performance than would crossing the horizontal meridian. As in Experiment 1, the accuracy data provided no evidence that crossing the vertical meridian leads to poorer performance than does crossing the horizontal

Table 2

Mean Percentage Correct, Mean Response Time (in Milliseconds), and Costs and Benefits in Relation to the Neutral Condition in Experiment 2

\begin{tabular}{lccccc}
\hline \multirow{2}{*}{\multicolumn{1}{c}{ Condition }} & \multicolumn{2}{c}{$\%$ Correct } & & \multicolumn{2}{c}{ RT } \\
\cline { 2 - 3 } \cline { 5 - 6 } \cline { 5 - 6 } & $M$ & Cost/Benefit & & $M$ & Cost/Benefit \\
\hline Valid & 76 & $6^{*}$ & & 720 & $100^{*}$ \\
Across-horizontal invalid & 62 & $-8 \dagger$ & & 823 & -3 \\
Across-vertical invalid & 62 & $-8 \dagger$ & & 861 & -41 \\
Diagonal invalid & 59 & $11 \neq$ & & 903 & $-83^{*}$ \\
Neutral & 70 & & & 820 & \\
\hline
\end{tabular}

Note-Positive numbers indicate benefits and negative numbers indicate costs in relation to the neutral condition. ${ }^{*} p<.10 . \quad+p<.05$. $\ddagger p<.01$. 
Table 3

Mean Response Time (in Milliseconds), and Costs and Benefits in Relation to the Neutral Condition in Experiment 3

\begin{tabular}{lcc}
\hline \multicolumn{1}{c}{ Condition } & RT & Cost/Benefit \\
\hline Valid & 488 & 5 \\
Across-horizontal invalid & 510 & $-17^{*}$ \\
Across-vertical invalid & 510 & $-17^{*}$ \\
Diagonal invalid & 519 & $-26 \dagger$ \\
Neutral & 493 & \\
\hline
\end{tabular}

Note-Positive numbers indicate benefits and negative numbers indicate costs in relation to the neutral condition. ${ }^{*} p<.05 . \quad{ }_{p}<.01$.

meridian. However, in the response time data, there was again a nonsignificant 38 -msec disadvantage for crossing the vertical meridian as opposed to the horizontal meridian. From these data, it might be tempting to conclude that although crossing the vertical meridian has no additional influence on accuracy, it does influence time to respond. However, we believe that once again, extreme caution must be exercised in interpreting the response time data in the across-vertical and across-horizontal invalid conditions, given that the accuracy rates were so low $(62 \%)$. In order to test more directly whether an additional cost in response time might be observed when crossing the vertical rather than the horizontal meridian, we conducted an additional experiment in which response times could more readily be interpreted.

\section{EXPERIMENT 3}

Experiment 3 was similar to Experiment 2 with two main exceptions. First, because we were interested in using response time as the primary dependent measure, the pattern mask that followed display of the target in Experiment 2 was not employed. The logic is that by increasing performance accuracy to near-ceiling level, the response times will be interpretable. Note that even without the mask, phosphor persistence could not increase the effective target duration because the stimuli were displayed in black on white (i.e., in reverse video). Second, we ran twice as many subjects (24) in Experiment 3 in order to increase the power of the study.

\section{Method}

Subjects. Twenty-four University of Alberta undergraduate students participated for credit toward their introductory psychology class. All subjects had normal or corrected-to-normal vision. None of the subjects had participated in Experiment 1 or 2.

Apparatus and Stimuli. The apparatus and stimuli were the same as those in Experiment 2.

Procedure. The procedure was identical to that in Experiment 2, except that no pattern mask followed display of the target. Instead, the fixation display appeared until a response was made. Following each response, the mask display appeared, serving as the between-trial display to indicate the four possible target locations (as had been the case in Experiment 2).

Each session began with the instructions along with several example trials, followed by 32 practice trials and one test block. The entire experiment lasted under $30 \mathrm{~min}$.

\section{Results}

As expected, overall accuracy was nearly perfect $(M=$ $98 \%$, range $=97 \%-98 \%$ across conditions). The primary dependent measure was therefore response time, as intended.

Response time analyses included only correct trials. In addition, outlier response times less than $100 \mathrm{msec}$, greater than 3,000 msec, or more than 3 standard deviations from the cell mean for that condition and that subject were discarded in order to reduce variability. In total, $1.75 \%$ of the potential data points were categorized as outliers.

Table 3 presents the mean response time for correct responses as a function of cue condition. A one-way omnibus analysis of variance showed a reliable effect of cuing $\left[F(4,92)=5.91, M S_{\mathrm{e}}=1,384, p<.001\right]$. As can be seen in Table 3, performance was again better when the target appeared at the cued location than when it appeared at any of the uncued locations.

The main question addressed in this experiment was whether performance would be worse in the acrossvertical invalid condition compared with the acrosshorizontal invalid condition when response time is used as the primary dependent measure. As can be seen in Table 3, performance in these two invalid conditions was virtually identical at $510 \mathrm{msec}\left(F<1, M S_{e}=252\right)$.

The costs and benefits associated with each of the other cue conditions in relation to the neutral condition are shown in Table 3. Response times in the neutral condition were once again intermediate between performance in the valid and invalid conditions.

\section{Discussion}

The pattern of results observed in Experiment 3, in which response time was the primary dependent measure, replicated in all essential forms the response accuracy results from Experiment 2. First, there was a robust effect of attentional orienting to an uninformative exogenous cue. Second, and most importantly, no difference was found for performance in the across-horizontal and acrossvertical invalid conditions. This result is inconsistent with the hypothesis that attentional orienting involves inhibiting the opposite horizontal visual hemifield (Hughes \& Zimba, 1985, 1987).

Finally, there was again an apparent distance effect, with response times for targets appearing in the diagonal invalid condition slower than those for targets appearing in the across-horizontal and across-vertical invalid conditions. This latter result is consistent with the gradient model of attentional orienting.

\section{GENERAL DISCUSSION}

In the present study, we investigated the spatial distribution of exogenous attention by using a stimulus discrimination paradigm. More specifically, we examined three main issues. First, we attempted to distinguish between 
the specific-location and general-region hypotheses concerning the spatial distribution of attention. Second, we investigated whether visual meridians play any role in the reorienting of visual attention following an invalid cue. Third, we examined the effect of the spatial distance between the cue and target on target discrimination.

Concerning the first issue, the results of the three experiments were clearly consistent with the specificlocation hypothesis and inconsistent with the generalregion hypothesis. The finding that performance was better at the cued location than at another location in the same visual hemifield (Experiments 1-3) and better at the cued location than at another location in the same visual quadrant (Experiment 1) strongly suggests that exogenous visual attention can be allocated to a specific location in the visual field, as proposed by theories that give visual attention a central role in feature binding and eyemovement control. It is important to note that our evidence supports the specific-location hypothesis even though targets were presented in an otherwise empty field. This finding is important because it has previously been suggested that evidence will only be found for the specificlocation hypothesis when the display field contains other nontarget stimuli (Hughes \& Zimba, 1987). The notion seems to be that when other markers are present, they will be used to help constrain the distribution of attention, particularly the distribution of locational inhibition. Instead, however, the current results suggest that exogenous attention can be allocated to a specific location even in the absence of other markers in the visual field.

The second issue that we addressed concerned whether the visual meridians play any role at all in the allocation of visuospatial attention. In Experiments 1 and 2, with response accuracy as the primary dependent measure, we found no effect of the visual meridians on that measure. Accuracy was equivalent for uncued locations within the cued quadrant and outside the cued quadrant (Experiment 1) and equivalent for uncued locations across the vertical and horizontal meridians (Experiment 2). There was a nonsignificant tendency in both experiments for response times to be longer when the target appeared at a location across the vertical meridian from the cued location than when the target appeared across the horizontal meridian. However, these response times were difficult to interpret in Experiment 1 because of a positional confound, and in both experiments because of high error rates. In Experiment 3, we examined response times more directly and with more statistical power and found no evidence for a meridian effect. The observed difference between the diagonal condition and the other two invalid conditions in all three experiments suggests that we do have the sensitivity to detect differences in the invalid conditions. These results strongly suggest that when exogenous attention is deployed in a stimulus discrimination task, the primary visual meridians play no significant role.

Taken together, our findings of better performance at the specific cued location compared with other locations in the same visual quadrant or hemifield, along with our findings that visual meridians played no role in invalid. trials, calls into question any version of Hughes and Zimba's $(1985,1987)$ meridian boundary model for exogenous attention. However, our findings with respect to exogenous attention do not necessarily imply that the general-region hypothesis would not hold in endogenous attentional paradigms. All we can conclude from our experiments is that exogenous attention does not appear to be constrained by the visual meridians. On the other hand, we note that several recent experiments call into question the viability of the meridian boundary model for paradigms engaging the endogenous attention system as well. For example, McCormick and Klein (1990; also Klein \& McCormick, 1989) have recently provided evidence that the meridian effects reported by Hughes and Zimba may be due to an artifact in their paradigm. Specifically, it appears that Hughes and Zimba induced their subjects to attend equivalently to the two target locations tested in the cued hemifield (1985) and quadrant (1987). Finally, there is evidence in a simple detection experiment of the type used by Hughes and Zimba that subjects can endogenously attend to a specific location within a region defined by a major meridian (Downing \& Pinker, 1985). Therefore, the evidence for the meridian boundary model is weak at best (see also Egly \& Homa, 1991).

The third issue that we addressed concerned the spatial distribution of exogenous visual attention. The results of the three experiments reported here are best characterized as a performance gradient over space. That is, performance was best at the cued location, and it dropped off as distance from the cued location increased. We believe that the most parsimonious account of this performance gradient is to assume that it reflects a similar underlying attentional gradient. On this view, a peak in the attentional gradient forms over the cued location (in an internal representation of visual space), and attention diminishes as spatial distance from this location increases. Because the rate of processing a stimulus at a given location is an increasing function of the distance of the location from the gradient peak, data-limited stimuli will be identified less accurately, and resource-limited stimuli will be identified less rapidly, as distance from the peak increases. Further, we assume that in the neutral condition, the resource gradient is flat, whereas in the cue conditions, the peak in the gradient forms by removing attentional resources (inhibiting) locations away from the peak and increasing resources (facilitating) locations at the peak (Henderson, 1991). In this way, benefits in performance are observed at the cued location, while costs are observed at uncued locations.

An alternative account of the effects of spatial distance on performance involves the suggestion that attention must be reoriented following an invalid cue, and that the time to reorient attention is a function of distance. Such an account would be consistent with any version of the spotlight view, such as the zoom-lens model (Eriksen \& St. James, 1986; Eriksen \& Webb, 1989), in which the spotlight must travel through an analogue representation 
of space as it is reoriented (see, e.g., Shulman, Remington, \& McLean, 1979; Tsal, 1983). In fact, Egly and Homa (1991) have recently proposed this type of account to explain the effects of spatial distance in a series of experiments similar to those reported here. However, we believe that there are a number of reasons for preferring the gradient model over an analogue-movement spotlight model. First, at this time there is very little empirical evidence that analogue movements of attention occur (see, e.g., Murphy \& Eriksen, 1987; Remington \& Pierce, 1984; Yantis, 1988). Second, one of the main motivations leading Egly and Homa (1991) to propose an analogue movement model was their finding that performance was worse for a same-quadrant invalid location than for a different-quadrant invalid location (Experiment 7; see also Tassinari et al., 1987). Because of this effect, Egly and Homa (1991) suggested that it would not be parsimonious to propose a gradient with such an unusual shape. However, we have found no such effect in six experiments (five experiments in Henderson, 1991, and Experiment 1 here). At this point, we are not sure how to account for the discrepancy in the results, but given that we cannot replicate their effect, we feel that the gradient model offers the most parsimonious account of the general pattern of data from the exogenous cuing paradigm.

\section{REFERENCES}

Abrams, R. A., Meyer, D. E., \& Kornblum, S. (1989). Speed and accuracy of saccadic eye movements: Characteristics of impulse variability in the oculomotor system. Joumal of Experimental Psychology: Human Perception \& Performance, 15, 529-543.

Bouma, H. (1971). Visual recognition of isolated lower-case letters. Vision Research, 11, 459-474.

Briand, K., \& Klein, R. M. (1987). Is Posner's "beam" the same as Treisman's "glue"?: On the relation between visual orienting and feature integration theory. Joumal of Experimental Psychology: Human Perception \& Performance, 13, 228-241.

DownING, C. J. (1988). Expectancy and visuo-spatial attention: Effects on perceptual quality. Journal of Experimental Psychology: Human Perception \& Performance, 14, 188-202.

Downing, C. J., Pinker, S. (1985). The spatial structure of visual attention. In M. I. Posner \& O. S. M. Marin (Eds.), Attention and performance $X I$ (pp. 171-188). Hillsdale, NJ: Erlbaum.

EGLY, R., \& HomA, D. (1991). Reallocation of visual attention. Journal of Experimental Psychology: Human Perception \& Performance, 17, 142-159.

ErIKsen, C. W., ST. James, J. D. (1986). Visual attention within and around the field of focal attention: A zoom lens model. Perception \& Psychophysics, 40, 225-240.

ErIKSEN, C. W., Webb, J. M. (1989). Shifting of attentional focus within and about a visual display. Perception \& Psychophysics, 45, 175-183.

ERIKSEN, C. W., \& YEH, Y. (1985). Allocation of attention in the visual field. Joumal of Experimental Psychology: Human Perception \& Performance, 11, 583-597.

HENDERSON, J. M. (1988). Visual attention and the acquisition of extrafoveal information during eye fixations. Unpublished doctoral dissertation, University of Massachusetts, Amherst.

Henderson, J. M. (1991). Stimulus discrimination following covert attentional orienting to an exogenous cue. Journal of Experimental Psychology: Human Perception \& Performance, 17, 91-106.

Henderson, J. M. (1992). Visual attention and eye movement control during reading and scene perception. In K. Rayner (Ed.), Eye movements and visual cognition: Scene perception and reading (pp. 260283). New York: Springer-Verlag.
Henderson, J. M. (in press). The interface between perception and action: Evidence from eye movement control. In S. Davis \& K. Aikens (Eds.), Vancouver studies in cognitive science: Vol. 4. Problems in perception. Oxford: Oxford University Press.

Henderson, J. M., \& Ferreira, F. (1990). The effect of foveal processing difficulty on the perceptual span in reading: Implications for attention and eye-movement control. Joumal of Experimental Psychology: Learning, Memory, \& Cognition, 16, 417-429.

Henderson, J. M., Pollatsek, A., \& Rayner, K. (1987). Effects of foveal priming and parafoveal preview on object identification. Journal of Experimental Psychology: Human Perception \& Performance, 13, 449-463.

Henderson, J. M., Pollatsek, A., \& Rayner, K. (1989). Covert visual attention and extrafoveal information use during object identification. Perception \& Psychophysics, 45, 196-208.

Hughes, H. C., \& Zimba, L. D. (1985). Spatial maps of directed visual attention. Joumal of Experimental Psychology: Human Perception \& Performance, 11, 409-430.

Hughes, H. C., ZimbA, L. D. (1987). Natural boundaries for the spread of directed visual attention. Neuropsychologia, 2, 5-18.

JAMES, W. (1890). The principles of psychology (Vol. 1). Cambridge, MA: Harvard University Press.

JONIDES, J. (1981). Voluntary versus automatic control over the mind's eye's movement. In J. Long \& A. Baddeley (Eds.), Attention and performance IX (pp. 187-203). Hillsdale, NJ: Erlbaum.

KINSBOURNE, M. (1987). Mechanisms of unilateral neglect. In M. Jeannerod (Ed.), Neurophysiological and neuropsychological aspects of spatial neglect. New York: North-Holland.

Klein, R., Kingstone, A., \& Pontefract, A. (1992). Orienting of visual attention. In $\mathrm{K}$. Rayner (Ed.), Eye movements and visual cognition: Scene perception and reading. New York: Springer-Verlag.

Klein, R., McCoRmick, P. (1989). Covert visual orienting: Hemifield activation can be mimicked by zoom lens and midiocation placement strategies. Acta Psychologica, 70, 235-250.

LABerge, D., \& Brown, V. (1989). Theory of attentional operations in shape identification. Psychological Review, 96, 101-124.

MADDEN, D. J. (1992). Selective attention and visual search: Revision of an allocation model and application to age differences. Joumal of Experimental Psychology: Human Perception \& Performance, 18, 821-836.

MAYLOR, E. A. (1985). Facilitatory and inhibitory components of orienting in visual space. In M. I. Posner \& O. S. M. Marin (Eds.), Attention and performance XI (pp. 189-204). Hillsdale, NJ: Erlbaum.

McCormick, P. A., \& KLEIN, R. (1990). The spatial distribution of attention during covert visual orienting. Acta Psychologica, 75, 225-242.

MorRISON, R. E. (1984). Manipulation of stimulus onset delay in reading: Evidence for parallel programming of saccades. Journal of Experimental Psychology: Human Perception \& Performance, 10, 667-682.

Murphy, T. D., \& Eriksen, C. W. (1987). Temporal changes in the distribution of attention in the visual field in response to precues. Perception \& Psychophysics, 42, 576-586.

MÜller, H. J., \& RABBITT, P. M. A. (1989). Reflexive and voluntary orienting of visual attention: Time course of activation and resistance to internuption. Joumal of Experimental Psychology: Human Perception \& Performance, 15, 315-330.

NakayAma, K., MACKEBEN, M. (1989). Sustained and transient components of focal visual attention. Vision Research, 29, 1631-1647.

Pollatsek, A., Rayner, K. (1990). Eye movements and lexical access in reading. In D. A. Balota, G. B. Flores d'Arcais, \& K. Rayner (Eds.), Comprehension processes in reading (pp. 143-163). Hillsdale, NJ: Erlbaum.

PoSNer, M. I. (1980). Orienting of attention. Quarterly Journal of Experimental Psychology, 32, 3-25.

Posner, M. I., \& Cohen, Y. (1984). Components of visual orienting. In H. Bouma \& D. Bowhuis (Eds.), Attention and performance $X$ (pp. 531-556). Hillsdale, NJ: Erlbaum.

Posner, M. I., Snyder, C. R. R., \& Davidson, B. J. (1980). Attention and the detection of signals. Journal of Experimental Psychology: General, 109, 160-174.

Rafal, R. D., Calabresi, P. A., Brennan, C. W., \& Sciolto, T. K. 
(1989). Saccadic preparation inhibits reorienting to recently attended locations. Journal of Experimental Psychology: Human Perception \& Performance, 15, 673-685.

Rayner, K., Slowiaczek, M. L., Clifton, C., \& Bertera, J. H. (1983). Latency of sequential eye movements: Implications for reading. Journal of Experimental Psychology: Human Perception \& Performance, 9, 912-922.

Remington, R., \& Pierce, L. (1984). Moving attention: Evidence for time-invariant shifts of visual selective attention. Perception \& Psychophysics, 35, 393-399.

Reuter-Lorenz, P. A., Kinsbourne, M., \& Moscovitch, M. (1990). Hemispheric control of spatial attention. Brain \& Cognition, 12 , 240-266.

Rizzolatti, G., Riggio, L., Dascola, I., \& Umiltà, C. (1987). Reorienting of attention across the horizontal and vertical meridians: Evidence in favor of a premotor theory of attention. Neuropsychologia, $25,31-40$.

Shulman, G. L., Remington, R. W., \& Mclean, J. P. (1979). Moving attention through visual space. Journal of Experimental Psychology: Human Perception \& Performance, 5, 522-526.

Shulman, G. L., Wilson, J., \& Sheehy, J. B. (1985). Spatial determinants of the distribution of attention. Perception \& Psychophysics, $37,59-65$.
Tassinari, G., Aglioti, S., Chelazzi, L., Marzi, C. A., \& BerlucCHI, G. (1987). Distribution in the visual field of the costs of voluntary allocated attention and of the inhibitory after-effects of covert orienting. Neuropsychologia, 25, 55-71.

Treisman, A., \& Gelade, G. (1980). A feature integration theory of attention. Cognitive Psychology, 12, 97-136.

TsaL, Y. (1983). Movements of attention across the visual field. Journal of Experimental Psychology: Human Perception \& Performance, 9, 523-530.

TSAL, Y. (1989). Do illusory conjunctions support the feature integration theory? A critical review of theory and findings. Journal of Experimental Psychology: Human Perception \& Performance, 15, 394-400.

Yantis, S. (1988). On analog movements of visual attention. Perception \& Psychophysics, 43, 203-206.

YANTIS, S., \& JONIDES, J. (1984). Abrupt visual onsets and selective attention: Evidence from visual search. Joumal of Experimental Psychology: Human Perception \& Performance, 10, 601-621.

(Manuscript received May 4, 1992; revision accepted for publication August 5, 1992.) 